\title{
Assessment of knowledge and attitude regarding use of contraceptive methods among women at selected rural area, Ambala, Haryana, India
}

\author{
Amandeep Kaur*, Baljinder Kaur, Abhishek Gupta, Amardeep Singh
}

Department of Nursing, M. M. College of Nursing, Mullana, Ambala, India

Received: 25 September 2015

Revised: 05 October 2015

Accepted: 29 October 2015

\section{*Correspondence:}

Amandeep Kaur,

E-mail: kaur.aman002@gmail.com

Copyright: ( ) the author(s), publisher and licensee Medip Academy. This is an open-access article distributed under the terms of the Creative Commons Attribution Non-Commercial License, which permits unrestricted non-commercial use, distribution, and reproduction in any medium, provided the original work is properly cited.

\section{ABSTRACT}

Background: Family planning refers to practices that help individuals and couples to attain certain objectives like to avoid unwanted births. The objectives of study were to assess the knowledge and attitude regarding use of contraceptive methods among women. Also to find out correlation between knowledge and attitude scores regarding use of contraceptive methods among women and to determine association of level of knowledge and attitude regarding use of contraceptive methods among women with selected demographic variables.

Methods: The research approach adopted for the study was quantitative research approach. The present study was carried out on the 200 women of reproductive age of village Rajouli, Ambala, Haryana selected by purposive sampling technique. The tools used for data collection were structured knowledge questionnaire and attitude scale (3 point likert's scale). Validity was ensured through consultation with the experts and reliability of knowledge questionnaire was established using Kuder's Richardson-20 formula whereas of attitude scale by split half method which is fund to be $(r=0.82)$ and $(r=0.80)$ respectively. Formal permission from sarpanch of village Rajouli.

Results: Descriptive and inferential statistics were used to analyze the data. Findings of study revealed that more than half $(51.5 \%)$ of the women were in the age group of 25-31 years. Results further illustrated that (38\%) of women had average knowledge and (44\%) moderately favorable attitude regarding use of contraceptive methods. There was significant association of level of knowledge of women with income per month (44.7), type of family (16.2), gap between first and second child (16.9), source of information about contraceptives (14.1) whereas level of attitude of women was found to be statistically significant with age (13.6), educational status (89.5), occupational status (20.2), family income per month (17.2), duration of marriage (19.7), number of children (17.9). There was significant positive correlation between knowledge and attitude scores as evident by computed ' $r$ ' value of 0.13 .

Conclusions: Hence, there is a need to create awareness among women of reproductive age group regarding contraceptive methods.

Keywords: Knowledge, Attitude, Contraceptive methods, Women

\section{INTRODUCTION}

Contraception means preventing the union of the sperm and ovum, suppressing ovulation or interfering with implantation of the fertilized ovum in the uterus. ${ }^{1}$
Family planning should be made accessible to every woman and man who needs it so that they can exercise their reproductive health rights, according to which everyone should be able to freely and responsibly decide how many children to have and when (that is, children "By Choice, and not by Chance"). ${ }^{2}$ 
Current projections show a continued increase in population with the global population expected to reach between 7.5 and 10.5 billion by $2050 .^{3}$

India's population as per 2011 census was 1.21 billion, second only to china in the world. The total fertility rate in India has declined from 6.0 in 1951 to 2.6 in 2009 but decline is not consistent in all the states. While 14 states have already achieved the replacement level, 12 states have Total Fertility Rate between 2.1 and 3 and 9 states (Bihar, U.P, Rajasthan, M.P, Jharkhand, Chhattisgarh, Meghalaya, Nagaland, D\&N Haveli) have Total Fertility Rate more than $3 .^{4}$

According to the National Family Health Survey conducted in India the most widely used method of family planning is female sterilization, which is accepted by $67 \%$ of current users as against male sterilization of $3 \%$. Women in developing countries are either under collective decision making with their partners or completely rely on the male partners decision on issues that affect their reproductive live. Identifying the major barriers of married women's decision-making power on contraceptive use has significant relevance for planning contextually appropriate family planning interventions. ${ }^{5}$

\section{METHODS}

A quantitative research approach with the descriptive survey design was adopted for the study. The study was conducted at Rajouli village of Ambala District Haryana. Study comprised of 200 married women of reproductive age who fulfill the inclusion criteria. Sample was selected by purposive sampling technique.

The data was collected by using structured knowledge questionnaire and three point Likert's scale. Structured knowledge questionnaire comprised of demographic variables and 30 knowledge items in multiple choice question related to Concept and Definitions, need, types, advantages, disadvantages and special instructions while using contraceptive methods. The attitude scale consisted of 20 items regarding contraceptive method. It covered the areas pertaining to contraceptive methods in term of concept of conception and contraception, need for contraception, types of contraceptive methods and their use, advantages, disadvantages and belief about contraceptive method. The attitude scale consists of 10 positive and 10 negative statements scattered randomly. Data was collected using paper pencil technique. The content validity of the tools was established by nine experts including four nursing experts in the field of Obstetrics and Gynecology, three from Medical-Surgical Nursing and two experts from Community Health Nursing Necessary suggestions were incorporated into tool. The tool translated in Hindi and verified by Hindi expert for appropriateness of language. Try-out was done on twenty women of Mullana village of Ambala district Haryana. The reliability coefficient for the structured knowledge questionnaire was calculated by using Kuder
Richardson-20 formula which was found to be 0.82 and for attitude scale by using split half method which was found to be 0.80 .

Formal approval was obtained from Sarpanch of Rajouli of Ambala District, Haryana. Data was collected on March 2015. Self-introduction \& introduction to the nature of the study were given to the selected samples. To obtain free $\&$ frank response, the participants of the study were assured about the confidentiality of their response. Written consent was taken from each participant. It took $45-50$ minutes to collect data. The data were analysed and interpreted as per the objectives. Descriptive and inferential statistics were used for the data analysis.

\section{RESULTS}

The data were analysed and interpreted as per the objectives. Descriptive and inferential statistics were used for the data analysis. Results revealed that more than half $(51.5 \%)$ of the women were in the age group of 25-31 years followed by $(23 \%)$ of women were in the age group of 32-38 years. Nearly half $(48 \%)$ of the women were educated up to secondary class. Majority of women $(82.5 \%)$ were homemaker followed by $(7 \%)$ of women were private employed. Forty percent $(40 \%)$ of women had monthly family income Rs 5001 to 10,000 whereas most of the $(53 \%)$ of women belonged to nuclear family. More than half $(56.5 \%)$ of the women were Sikh and thirty eight percent $(38 \%)$ of women were Hindu. Most of the $(45.5 \%)$ of women had 6-10 years duration of marriage and $(59.5 \%)$ of the women had two living child. One third $(36.5 \%)$ of women had 1 year's gap between first and second child and (43\%) of women had television as source of information about contraceptives. Forty two percent $(42 \%)$ of the women using condom as contraceptive method, followed by $(29 \%)$ of the women are using $\mathrm{Cu} \mathrm{T}$ as contraceptive method, followed by $(23 \%)$ of the women are using tubectomy as contraceptive method and (16\%) of the women are using contraceptive pills as contraceptive method (Table 1).

\section{Description of knowledge scores}

Study findings further showed that thirty eight percent $(38 \%)$ of women had average knowledge followed by $(32.5 \%)$ of women had good knowledge, (19\%) of women had below average knowledge and only $(10.5 \%)$ of women had excellent knowledge regarding use of the contraceptive methods (Figure 1).

\section{Description of attitude scores}

Results illustrated that forty four percent (44\%) of women had moderate attitude followed by (37\%) of women had unfavourable attitude, and only (19\%) of women had favourable attitude regarding use of contraceptive methods (Figure 2). 
Table 1: Frequency \& percentage distribution of women by their selected demographic variables $(\mathrm{N}=\mathbf{2 0 0})$.

\begin{tabular}{|c|c|c|c|}
\hline No. & Demographic Variables & $\mathbf{F}$ & $(\%)$ \\
\hline 1. & Age (In Years) & & \\
\hline A & $18-24$ & 25 & 12.5 \\
\hline B & $25-31$ & 103 & 51.5 \\
\hline $\mathrm{C}$ & $32-38$ & 46 & 23 \\
\hline $\mathrm{D}$ & $39-45$ & 26 & 13 \\
\hline 2. & Educational Status & & \\
\hline A & Primary & 40 & 20 \\
\hline B & Secondary & 96 & 48 \\
\hline $\mathrm{C}$ & Senior Secondary & 53 & 26.5 \\
\hline $\mathrm{D}$ & Graduate \& Above & 11 & 5.5 \\
\hline 3. & Occupation Status & & \\
\hline A & Self Employed & 12 & 6 \\
\hline B & Government Employee & 9 & 4.5 \\
\hline $\mathrm{C}$ & Private Employee & 14 & 7 \\
\hline $\mathrm{D}$ & Homemaker & 165 & 82.5 \\
\hline 4. & Family Income Per Month (In Rs.) & & \\
\hline A & $\leq 5000$ & 30 & 15 \\
\hline B & $5001-10,000$ & 80 & 40 \\
\hline $\mathrm{C}$ & $10,001-15,000$ & 60 & 30 \\
\hline $\mathrm{D}$ & $\geq 15,000$ & 30 & 15 \\
\hline 5. & Type Of Family & & \\
\hline A & Nuclear Family & 106 & 53 \\
\hline B & Joint Family & 89 & 44.5 \\
\hline $\mathrm{C}$ & Extended Family & 5 & 2.5 \\
\hline 6. & Religion & & \\
\hline A & Hindu & 76 & 38 \\
\hline B & Muslim & 7 & 3.5 \\
\hline $\mathrm{C}$ & Christian & 4 & 2 \\
\hline $\mathrm{D}$ & Sikh & 113 & 56.5 \\
\hline 7. & Duration Of Marriage & & \\
\hline A & $0-5$ Years & 66 & 33 \\
\hline B & 6-10 Years & 91 & 45.5 \\
\hline $\mathrm{C}$ & 11-15 Years & 23 & 11.5 \\
\hline $\mathrm{D}$ & $>15$ Years & 20 & 10 \\
\hline 8. & Number Of Children & & \\
\hline A & One & 57 & 28.5 \\
\hline B & Two & 119 & 59.5 \\
\hline $\mathrm{C}$ & Three & 22 & 11 \\
\hline $\mathrm{D}$ & $>3$ & 2 & 1 \\
\hline 9. & Gap Between First And Second Child & & \\
\hline A & 1 Years & 73 & 36.5 \\
\hline B & 2 Years & 71 & 35.5 \\
\hline $\mathrm{C}$ & 3 Years & 45 & 22.5 \\
\hline $\mathrm{D}$ & 4 Years & 11 & 5.5 \\
\hline 10 & Source Of Information About Contraceptives & & \\
\hline A & Television & 86 & 43 \\
\hline B & Newspaper/Family Members/Friends & 54 & 27 \\
\hline $\mathrm{C}$ & Health Personnel & 60 & 30 \\
\hline 11 & Contraceptive Method Used & & \\
\hline A & Condom & 84 & 42 \\
\hline B & Tubectomy & 46 & 23 \\
\hline $\mathrm{C}$ & $\mathrm{Cu}-\mathrm{T}$ & 38 & 29 \\
\hline $\mathrm{D}$ & Contraceptive Pills & 32 & 16 \\
\hline
\end{tabular}


Table 2: Chi square value showing association of level of knowledge regarding use of contraceptive method among women with selected demographic variables $(\mathrm{N}=200)$.

\begin{tabular}{|c|c|c|c|c|c|c|c|c|}
\hline & \multicolumn{8}{|c|}{ Knowledge Score } \\
\hline No. & Demographic Variables & $\begin{array}{l}\text { Very } \\
\text { Good } \\
\mathbf{7 0 \%} \\
(\mathrm{N}=\mathbf{2 0})\end{array}$ & $\begin{array}{l}\text { Good } \\
\begin{array}{l}(60-70 \%) \\
(N=65)\end{array}\end{array}$ & $\begin{array}{l}\text { Average } \\
\qquad \begin{array}{l}(50-60 \%) \\
(\mathrm{N}=76)\end{array}\end{array}$ & $\begin{array}{c}\text { Below } \\
\text { Average } \\
<\mathbf{5 0 \%} \\
(\mathrm{N}=\mathbf{3 9})\end{array}$ & $x^{2}$ & Df & $\begin{array}{l}\text { Table } \\
\text { Value }\end{array}$ \\
\hline 1. & Age (In Years) & & & & & & & \\
\hline A & $18-24$ & $3(15)$ & $10(15.3)$ & $6(7.8)$ & $6(15.3)$ & \multirow{4}{*}{$6.83^{\text {ns }}$} & \multirow{4}{*}{9} & \multirow{4}{*}{$\begin{array}{l}16.9 \\
1\end{array}$} \\
\hline B & $25-31$ & $9(45)$ & $32(49.2)$ & $39(51.3)$ & $23(58.9)$ & & & \\
\hline $\mathrm{C}$ & $32-38$ & $5(25)$ & $17(26.1)$ & $17(22.3)$ & $7(17.9)$ & & & \\
\hline $\mathrm{D}$ & $39-45$ & $3(15)$ & $6(9.2)$ & $14(18.4)$ & $3(7.6)$ & & & \\
\hline 2. & \multicolumn{5}{|l|}{ Educational Status } & \multirow{5}{*}{$14.5^{\text {ns }}$} & \multirow{5}{*}{9} & \multirow{5}{*}{16.91} \\
\hline A & Primary & $3(15)$ & $12(18.4)$ & $16((210$ & $9(23)$ & & & \\
\hline B & Secondary & $6(30)$ & $30(46.1)$ & $35(46)$ & $25(64.1)$ & & & \\
\hline $\mathrm{C}$ & Senior Secondary & $9(45)$ & $17(26.1)$ & $22(28.9)$ & $5(12.8)$ & & & \\
\hline $\mathrm{D}$ & Graduate \& Above & $2(10)$ & $6(9.2)$ & $3(3.9)$ & $0(0)$ & & & \\
\hline 3. & \multicolumn{5}{|l|}{ Occupation Status } & \multirow{5}{*}{$8.96^{\mathrm{ns}}$} & \multirow{5}{*}{9} & \multirow{5}{*}{16.91} \\
\hline A & Self Employed & $0(0)$ & $5(7.6)$ & $5(6.5)$ & $2(5.1)$ & & & \\
\hline B & Government Employee & $3(15)$ & $1(1.5)$ & $4(5.2)$ & $1(2.5)$ & & & \\
\hline $\mathrm{C}$ & Private Employee & $2(10)$ & $5(7.6)$ & $5(6.5)$ & $2(2.5)$ & & & \\
\hline $\mathrm{D}$ & Homemaker & $15(75)$ & $54(83)$ & $62(82.6)$ & $34(87.5)$ & & & \\
\hline 4. & \multicolumn{5}{|c|}{ Family Income Per Month(In Rs) } & \multirow{5}{*}{$44.7^{*}$} & & \\
\hline A & $\leq 5000$ & $2(10)$ & $5(7.6)$ & $8(10.5)$ & $15(38.4)$ & & \multirow{4}{*}{9} & \multirow{4}{*}{16.91} \\
\hline B & $5001-10,000$ & $1(05)$ & $23(35.3)$ & $38(50)$ & $18(46.1)$ & & & \\
\hline $\mathrm{C}$ & $10,001-15,000$ & $12(60)$ & $24(36.9)$ & $19(25)$ & $5(12.8)$ & & & \\
\hline $\mathrm{D}$ & $\geq 15,000$ & $5(10)$ & $13(20)$ & $11(14.4)$ & $1(2.5)$ & & & \\
\hline 6. & \multicolumn{5}{|l|}{ Type Of Family } & \multirow{4}{*}{$16.2^{*}$} & & \\
\hline A & Nuclear Family & $9(45)$ & $34(52.3)$ & $43(56.5)$ & $20(51.2)$ & & \multirow{3}{*}{6} & \multirow{3}{*}{12.52} \\
\hline B & Joint Family & $8(40)$ & $31(47.6)$ & $31(40.7)$ & $19(48.7)$ & & & \\
\hline $\mathrm{C}$ & Extended Family & $3(15)$ & $0(0)$ & $2(2.6)$ & $0(0)$ & & & \\
\hline 7. & Religion & & & & & & & \\
\hline A. & Hindu & $8(40)$ & $27(41.5)$ & $25(32.8)$ & $16(41)$ & & & \\
\hline B. & Muslim & $0(0)$ & $0(0)$ & $7(9.2)$ & $0(0)$ & $12^{\mathrm{ns}}$ & 0 & 1600 \\
\hline C. & Christian & $0(0)$ & $1(1.5)$ & $2(2.6)$ & $1(2.5)$ & $13^{-3}$ & 9 & 16.91 \\
\hline D. & Sikh & $12(60)$ & $37(56.9)$ & $42(55.2)$ & $22(56.4)$ & & & \\
\hline 8. & Duration Of Marriage & & & & & & & \\
\hline A & $0-5$ Years & $5(25)$ & $24(36.9)$ & $24(31.5)$ & $13(33.3)$ & & & 16.91 \\
\hline B & 6-10 Years & $6(39)$ & $32(49.2)$ & $33(43.4)$ & $20(51.2)$ & $13.6^{\mathrm{ns}}$ & . & \\
\hline $\mathrm{C}$ & 11-15 Years & $5(25)$ & $5(7.6)$ & $12(15.7)$ & $1(2,5)$ & & 9 & \\
\hline $\mathrm{D}$ & $>15$ years & $4(20)$ & $4(6.1)$ & $7(9.2)$ & $5(12.8)$ & & & \\
\hline 9 & Number Of Children & & & & & & & \\
\hline A & One & $7(35)$ & $20(30.7)$ & 21(27.6) & $9(23)$ & & & \\
\hline B & Two & $10(50)$ & $39(60)$ & $44(57.8)$ & $26(66.6)$ & $6.37^{\mathrm{ns}}$ & 0 & 1601 \\
\hline $\mathrm{C}$ & Three & $2(10)$ & $6(9.2)$ & $10(13.1)$ & $4(10.2)$ & & 9 & 10.91 \\
\hline $\mathrm{D}$ & $>3$ & $1(5)$ & $0(0)$ & $1(1.3)$ & $0(0)$ & & & \\
\hline 10 & Gap Between First And Se & Child & & & & & & \\
\hline A & 1 Years & $6(30)$ & $28(43)$ & $26(34.2)$ & $13(33.3)$ & & & \\
\hline $\mathrm{B}$ & 2 years & $3(15)$ & 20(30.7) & $33(43)$ & $15(38.4)$ & $16.9^{*}$ & 9 & 16.91 \\
\hline $\mathrm{C}$ & 3 Years & $7(35)$ & $13(20)$ & $15(19.7)$ & $10(25.6)$ & & 9 & 16.91 \\
\hline $\mathrm{D}$ & 4 Years & $4(20)$ & $4(6.1)$ & $2(2.6)$ & $1(2.5)$ & & & \\
\hline 11 & Source Of Information Ab & ontracept & & & & & & \\
\hline A & Television & $6(30)$ & $27(41.5)$ & $39(51.3)$ & $14(35.8)$ & $141^{*}$ & & \\
\hline B & $\begin{array}{l}\text { Newspaper/Family } \\
\text { Members/Friends }\end{array}$ & $10(50)$ & $18(27.6)$ & $11(4.4)$ & $15(38.4)$ & 14.1 & 6 & 12.52 \\
\hline
\end{tabular}




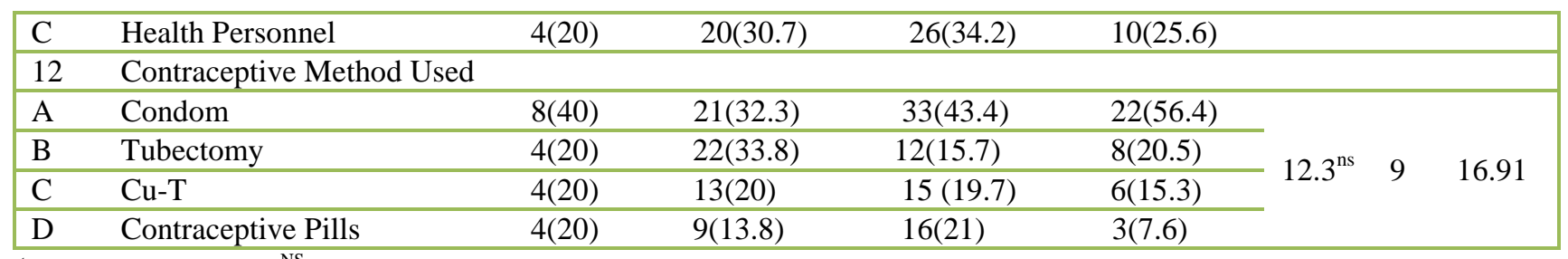

$(p<0.05) *$ Significant ${ }^{\mathrm{NS}} ;$ Non significant

Table 3: Chi square value showing association of level of attitude regarding use of contraceptive method among women with selected demographic variables $(\mathrm{N}=200)$.

\begin{tabular}{|c|c|c|c|c|c|c|c|}
\hline No. & Demographic Variables & $\begin{array}{cc}\text { Favourable } & \begin{array}{c}\text { A } \\
\\
\end{array} \\
\text { Un } \\
(41-60) \\
(n=38)\end{array}$ & $\begin{array}{l}\text { itude Score } \\
\text { derately } \\
\text { ivourable } \\
(\mathbf{3 1}-40) \\
(\mathbf{n}=\mathbf{8 8})\end{array}$ & $\begin{array}{l}\text { Favourable } \\
\begin{array}{c}(20-30) \\
(n=74)\end{array}\end{array}$ & $\mathrm{X}^{2}$ & Df & $\begin{array}{l}\text { Table } \\
\text { Value }\end{array}$ \\
\hline 1. & Age (In Years) & & & & & & \\
\hline A & $18-24$ & $9(23.6)$ & $10(22.7)$ & $5(6.7)$ & \multirow{4}{*}{$13.06^{*}$} & \multirow{4}{*}{3} & \multirow{4}{*}{12.52} \\
\hline B & $25-31$ & $15(39.4)$ & $47(53.4)$ & $39(52.7)$ & & & \\
\hline $\mathrm{C}$ & $32-38$ & $13(34.2)$ & $17(19.3)$ & $19(25.6)$ & & & \\
\hline $\mathrm{D}$ & $39-45$ & $1(5.2)$ & $14(15.9)$ & $11(14.8)$ & & & \\
\hline 2. & Educational Status & & & & & & \\
\hline A & Primary & $3(7.8)$ & $23(26.1)$ & 14948.9) & \multirow{4}{*}{$10.7^{*}$} & \multirow{4}{*}{3} & \multirow{4}{*}{12.52} \\
\hline $\mathrm{B}$ & Secondary & $4(10.5)$ & $30(34)$ & $60(81)$ & & & \\
\hline $\mathrm{C}$ & Senior Secondary & $25(65.7)$ & $28(31.8)$ & $0(0)$ & & & \\
\hline $\mathrm{D}$ & Graduate \& Above & $6(15.7)$ & $7(7.9)$ & $0(0)$ & & & \\
\hline 3. & Occupation Status & & & & & & \\
\hline A & Self Employed & $5(13.1)$ & $5(5.6)$ & $3(4)$ & \multirow{4}{*}{$8.96^{\mathrm{ns}}$} & \multirow{4}{*}{3} & \multirow{4}{*}{12.52} \\
\hline B & Government Employee & $2(5.2)$ & $6(6.8)$ & $0(00$ & & & \\
\hline $\mathrm{C}$ & Private Employee & $7(18.4)$ & $5(5.6)$ & $2(2.7)$ & & & \\
\hline $\mathrm{D}$ & Homemaker & $24(63.1)$ & $72(81.8)$ & 69(93.2) & & & \\
\hline 4. & Family Income Per Month & & & & & & \\
\hline A & $\leq 5000$ & $3(7.8)$ & $14(15.9)$ & 12(16.2) & \multirow{4}{*}{$3.68^{*}$} & \multirow{4}{*}{3} & \multirow{4}{*}{12.52} \\
\hline B & $5001-10,000$ & $10(26.3)$ & $39(44.3)$ & $33(44.5)$ & & & \\
\hline $\mathrm{C}$ & $10,001-15,000$ & $13(34.2)$ & $29(32.9)$ & $21(28.3)$ & & & \\
\hline $\mathrm{D}$ & $\geq 15,000$ & $12(31.5)$ & $6(6.8)$ & $8(16.8)$ & & & \\
\hline 5. & Type Of Family & & & & & & \\
\hline A & Nuclear Family & $15(29.4)$ & $54(61.3)$ & $38(51.3)$ & \multirow{3}{*}{$6.27^{*}$} & \multirow{3}{*}{2} & \multirow{3}{*}{9.48} \\
\hline $\mathrm{B}$ & Joint Family & $23(60.5)$ & $30(34)$ & $34(45.9)$ & & & \\
\hline $\mathrm{C}$ & Extended Family & $0(0)$ & $4(4.5)$ & $2(2.7)$ & & & \\
\hline 6. & Religion & & & & & & \\
\hline A. & Hindu & $10(26.1)$ & $33(37.5)$ & $32(43.2)$ & \multirow{4}{*}{$1.65^{*}$} & \multirow{4}{*}{3} & \multirow{4}{*}{12.52} \\
\hline B. & Muslim & $01(5.2)$ & $3(3.4)$ & $0(0)$ & & & \\
\hline C. & Christian & $00(0)$ & $1(1.1)$ & $1(1.3)$ & & & \\
\hline D. & Sikh & 27 & $51(57.9)$ & $41(55.4)$ & & & \\
\hline 7. & Duration Of Marriage & & & & & & \\
\hline A & $0-5$ Years & $11(28.9)$ & $35(39.7)$ & $17(22.9)$ & \multirow{4}{*}{$7.74^{\mathrm{NS}}$} & \multirow{4}{*}{3} & \multirow{4}{*}{12.52} \\
\hline $\mathrm{B}$ & 6-10 Years & $23(60.5)$ & $41(46.5)$ & $32(43.2)$ & & & \\
\hline $\mathrm{C}$ & 11-15 Years & $1(5.2)$ & $8(9.9)$ & $12(16.2)$ & & & \\
\hline $\mathrm{D}$ & $>15$ years & $1(5.2)$ & $4(4.5)$ & 13(17.4) & & & \\
\hline 8. & Number Of Children & & & & & & \\
\hline A & One & $9(23.6)$ & $36(40.9)$ & $10(13.4)$ & \multirow{3}{*}{$16.1^{*}$} & \multirow{3}{*}{3} & \multirow{3}{*}{12.52} \\
\hline $\mathrm{B}$ & Two & $25(65.7)$ & $44(50)$ & $52(61.9)$ & & & \\
\hline $\mathrm{C}$ & Three & $4(10.5)$ & $7(7.9)$ & $12(16.2)$ & & & \\
\hline
\end{tabular}




\begin{tabular}{|c|c|c|c|c|c|c|c|}
\hline D & $>3$ & $0(0)$ & $1(1.1)$ & $0(0)$ & & & \\
\hline 9. & Gap Between First $t$ & hild & & & & & \\
\hline A & 1 Years & $10(22.7)$ & $33(37.5)$ & $27(36.4)$ & \multirow{4}{*}{$0.441^{\mathrm{NS}}$} & \multirow{4}{*}{3} & \multirow{4}{*}{12.52} \\
\hline B & 2 years & $19(50)$ & $32(36.3)$ & $24(32.4)$ & & & \\
\hline $\mathrm{C}$ & 3 Years & $5(13.1)$ & $18(21.5)$ & $20(27)$ & & & \\
\hline D & 4 Years & $4(10.5)$ & $5(5.6)$ & $3(4)$ & & & \\
\hline 10 & \multicolumn{4}{|c|}{ Source Of Information About Contraceptives } & \multirow{5}{*}{$5.83^{\mathrm{NS}}$} & & \\
\hline A & Television & 17(44.7) & $47(53.4)$ & $26(35.1)$ & & \multirow{4}{*}{2} & \multirow{3}{*}{9.48} \\
\hline B & $\begin{array}{l}\text { Newspaper/Family } \\
\text { Members/Friends }\end{array}$ & $9(23.6)$ & 1618.1) & $26(35.1)$ & & & \\
\hline $\mathrm{C}$ & Health Personnel & $12(31.5)$ & $25(28.4)$ & $22(26.1)$ & & & \\
\hline 11 & Contraceptive Meth & & & & & & \\
\hline A & Condom & $15(39.8)$ & 3843.1) & $30(40.5)$ & \multirow{4}{*}{$0.73^{\mathrm{NS}}$} & \multirow{4}{*}{3} & \multirow{4}{*}{12.52} \\
\hline $\mathrm{B}$ & Tubectomy & $14(36.8)$ & $19(21.5)$ & $16(21.4)$ & & & \\
\hline $\mathrm{C}$ & $\mathrm{Cu}-\mathrm{T}$ & $5(5.6)$ & $8(20.4)$ & $16(21.4)$ & & & \\
\hline $\mathrm{D}$ & Contraceptive Pills & $4(4.5)$ & 13(14.7) & $12(16.2)$ & & & \\
\hline
\end{tabular}

$(p<0.05) *$ Significant ${ }^{\text {NS}} ;$ Non significant

\section{Correlation between knowledge and attitude of women}

Furthermore results revealed that coefficient of correlation between knowledge and attitude score is 0.13 , which shows positive correlation between knowledge and attitude of women regarding contraceptive method.

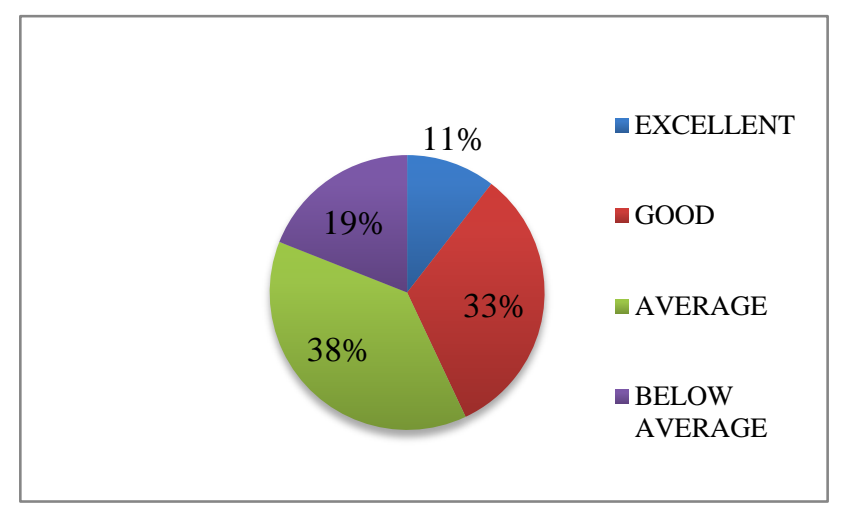

Figure 1: Percentage distribution of knowledge scores obtained by women.

Association of level of knowledge with selected demographic variables

Computed chi square value of level of knowledge regarding use of contraceptive method among women with selected demographic variables. The findings suggest that the computed chi square value of family income per month (44.7), type of family (16.2), gap between first and second child (16.9), source of information about contraceptives (14.1) were found to be statistically significant at 0.05 level of significance. It was found that level of knowledge depend on family income per month, type of family, gap between first and second child, source of information about contraceptive methods whereas the computed chi square value of age (6.83), educational status (14.5), occupational status
(8.96), religion (13.0), duration of marriage (13.6), number of children (6.37), contraceptive method (12.3) was found to be statistically non-significant (Table 2).

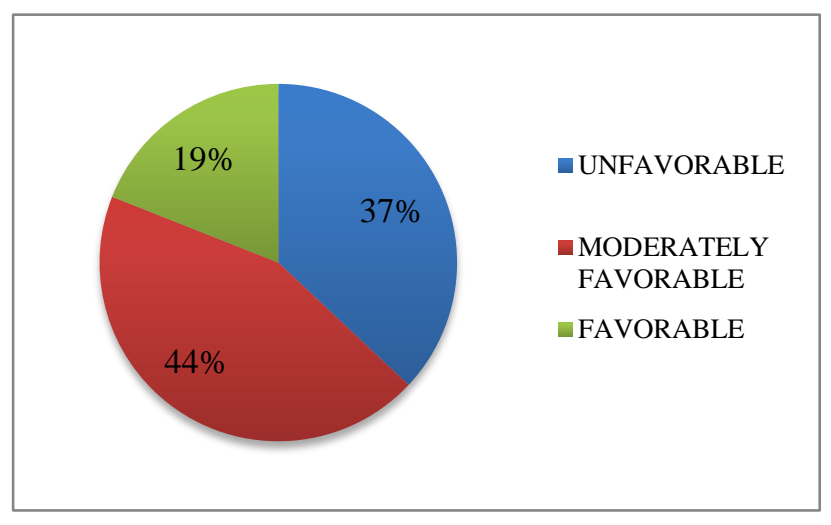

Figure 2: Percentage distribution of attitude scores obtained by women.

\section{Association of level of attitude with selected demographic variables}

Table 4 depicts computed chi square value of level of attitude with age (13.6), educational status (89.5), occupational status (20.2), family income per month (17.2), duration of marriage (19.7), number of children (17.9), was found to be statistically significant whereas with type of family (8.77), religion (5.93), gap between first and second child (7.25), source of information (7.67) and contraceptive method use (4.60) was found be statistically non-significant. This showed level of attitude of women regarding use contraceptive methods was dependent on the age, educational status, occupational status, family income per month, duration of marriage, number of children whereas independent on type of family, religion, gap between first and second child, source of information, contraceptive method to be used by women (Table 3 ). 


\section{DISCUSSION}

Findings of the study showed that $(38 \%)$ of women had average knowledge about contraceptive methods. These findings are consistent with the cross sectional study conducted at Moodu Alevoor village by Sonam Zangmu Sherpa et al where it was found that majority $(67.60 \%)$ had moderate knowledge. ${ }^{6}$

The study findings show that $(44 \%)$ of the women had moderately favourable attitude regarding use of contraceptive methods. These findings are consistent with the study conducted at Agroha village by N Saluja et al where positive attitude for contraception was shown by $198(79.2 \%)$ females and $158(63.2 \%)$ males. $^{7}$

Furthermore results revealed that level of knowledge found to be statistically significant with family income per month (44.7), type of family (16.2), source of information about contraceptives (14.1). These findings are consistent with findings of another study conducted among married women by Shabana Anjum et al where it was revealed that Socio demographic variable were significantly associated with existing knowledge and level of married women specially age at marriage, age at first child, occupation, income, education. ${ }^{8}$

The finding of the study has implications for nursing practice, nursing education, community health practice and nursing research. Health education can be conducted by the nursing personnel regarding use of contraceptive methods for reproductive age women as the number of women undergoing abortion due to unwanted pregnancy and mothers have limited knowledge, and unfavourable attitude about contraceptive methods. further studies can be conducted to explore the methods/strategies by which their knowledge and attitude can be improved/enhanced through many multiple researches are conducted by various nursing personnel at various level yet there is serious lack in disseminations of finding.

\section{ACKNOWLEDGEMENTS}

At the very outset, I would like to thank almighty for his presence experienced during the study. Our heartily thank to Mrs. Poonam Sheoran, Associate Professor, M. M. College of Nursing for their valuable suggestions and corrections.

Funding: No funding sources

Conflict of interest: None declared

Ethical approval: Ethical approval to conduct the study was obtained from Sarpanch of village Rajouli, Ambala, Haryana. Written informed consent was obtained from the study subjects regarding their willingness to participate in the research project

\section{REFERENCES}

1. Park K. Textbook of Preventive \& Social Medicine. $19^{\text {th }}$ ed. M/s Banarsidas Bhanot. 2008:389-391.

2. State of World Population 2012 (By Choice, not by Chance): http://www.unfpa.org/public/home/ publications/pid/12511.

3. World population available at: http://.www.wikipedia.org/wiki/World_population.

4. Ministry of health and family welfare programme year 2010-2011 NRHM 7-8 July 2011.

5. Stabilizing population; Ministry of information and broadcasting available atwww.rrtd.nic.in/population.

6. Sonam Zangmu Sherpa et al. knowledge, attitude, practice and preferences of contraceptive methods in udupi district, Karnataka. Journal of Family Reprod Health. 2013;7(3):115-20.

7. N Saluja, S Sharma, S Choudhary, D Gaur, S Pandey. Contraceptive Knowledge, Attitude and Practice among Eligible Couples of Rural Haryana. The Internet Journal of Health. 2009;12(1).

8. Anjum S. Knowledge of Contraceptives Methods and Appraisal of Health Education among Married Woman. International Journal of Science and Research (IJSR). 2014;3(3):584-90.

Cite this article as: Pattnaik T, Samal S, Behuria S. Obstetric admissions to the intensive care unit: a 5year review. Int J Reprod Contracept Obstet Gynecol 2015;4:1907-13. 